Proc. XII Int. School on Theoretical Physics - Symmetry and Structural Properties of Condensed Matter

\title{
Stiffness of Auxetic Foams
}

\author{
T. WIĘCEK* AND A. WASILEWSKI
}

Rzeszów University of Technology, al. Powstanców Warszawy 12, Rzeszów, Poland

An optical method is used for measurements of mechanical properties of foams. The method is based on analysis of diffraction pattern and does not require calibration. The measurements of the stiffness were performed for the conventional and auxetic foams. It was found that the stiffness of auxetic foams is several times greater than that of conventional foams.

DOI: 10.12693/APhysPolA.132.182

PACS/topics: $62.20 . \mathrm{dq}$

\section{Introduction}

Polyurethane foams are materials have long been found in every area of life. Foamed plastics are versatile. By modifying their cellular structure can be obtained the auxetic structure. Such materials are characterized by superior utility properties than traditional foam materials. Traditional polymer foams have the Poisson ratio from 0.1 to 0.4 .

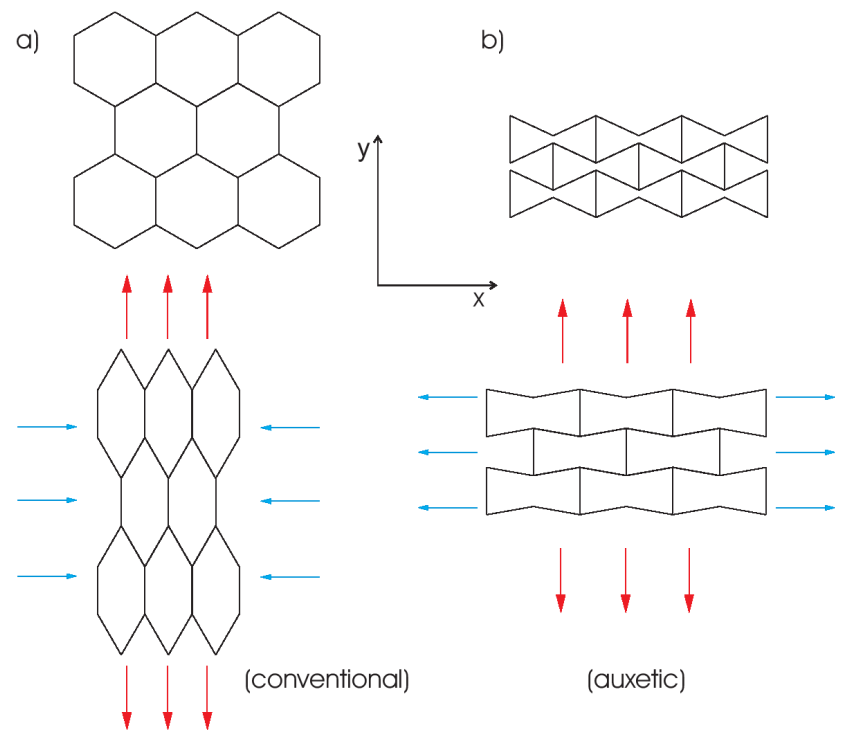

Fig. 1. Schematic diagram showing (a) a conventional honeycomb and (b) an auxetic honeycomb.

the Poisson ratio is defined as the ratio of the transverse deformation to the longitudinal deformation with an axial stress state. Auxetic materials display negative the Poisson ratio, i.e. they are not common ones. When stretched they become thicker perpendicularly to the applied force. Such materials are expected to have interesting mechanical properties, e.g. high energy absorption. Their structure can be of honeycomb form (Fig. 1). The

\footnotetext{
*corresponding author; e-mail: ftkwiece@prz.edu.pl
}

auxetic foams have negative the Poisson ratio values from -0.1 to -0.7 [1]. Foam materials with negative the Poisson were first produced by Lakes [2] and then by several others [3-7].

Conventional viscoelastic foams NVE65H manufactured by Natural Chemical Products Poland were compressed triaxially, in three orthogonal directions (Fig. 2) and next were put for $10 \mathrm{~min}$ to a container which had the temperature of $200^{\circ} \mathrm{C}$ [8]. Sample dimensions $a \times b \times c$ after compressions were $0.714 a \times 0.714 b \times 0.714 c$ according to the recommended literature [2-7]. The stiffness $k$ of a body is a measure of the resistance offered by an elastic body to deformation

$$
k=\frac{F}{\Delta l}
$$

where $F$ is the force applied on the body, $\Delta l$ is the displacement. The measurement of the stiffness were performed for the conventional and auxetic foams by using laser system.

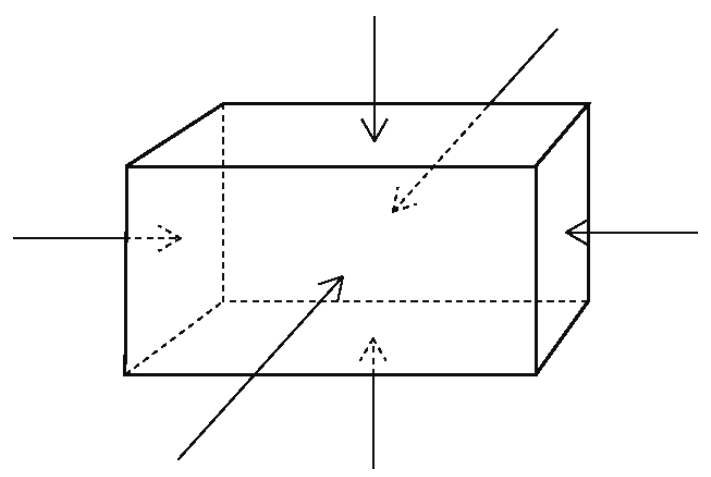

Fig. 2. Conventional foam is compressed triaxially.

\section{Experimental measurement system}

A laser beam falls on a very small slit between the reference edge 2 and the mirror 1 . We obtain the diffraction pattern on the screen S. Laser Compass 215 of $\lambda=532 \mathrm{~nm}$ has been used. The intensity distribution of the diffraction pattern is described by the Fraunhofer formula [9]: 


$$
I=I_{0}\left[\frac{\sin \left(\frac{\pi D}{\lambda f} x\right)}{\frac{\pi D}{\lambda f} x}\right]^{2},
$$

where $\lambda=0.532 \mu \mathrm{m}, f$ - the distance between the screen and the slit, $I_{0}$ - the light intensity at $x=0$, $D$ - the width of the slit, $x$ - the position of fringes.

One should point out that the proposed method is a non-contact and non-inertial one [9-13]. In the mechanics, and physics, Hooke's law of elasticity is an approximation which states that the extension of a spring is in a direct proportion with the load added to it as long as this load does not exceed the elastic limit.

\section{Results}

Our optical measurement method is based on a diffraction phenomena of light on a slit. The experimental system is shown in Fig. 3.

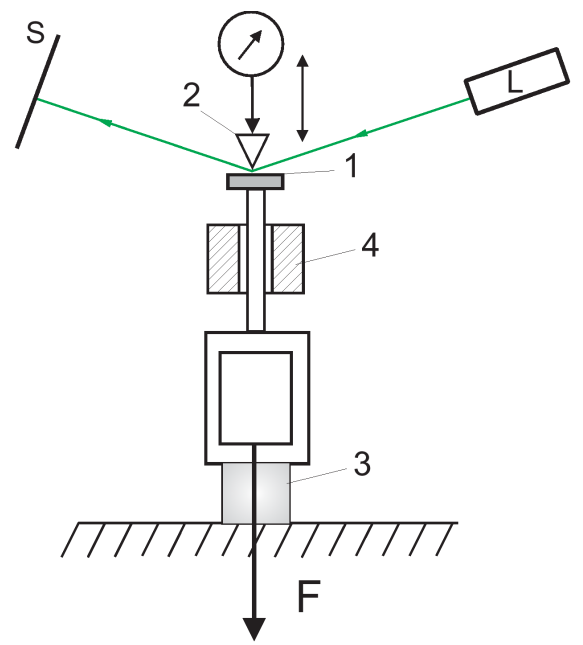

Fig. 3. Experimental measurement system: $\mathrm{S}-$ screen, L - laser, $\mathrm{F}$ - applied force, 1 - mirror, 2 - reference edge, 3 - auxetic or conventional foam, 4 — air bearing.

The laser beam is incident on the slit created by a reference edge and a moving mirror. The diffraction pattern is observed on the screen with a standard scale where dark fringes are of the same width as the slit. The position of the moving mirror can be measured by a sensor. The deformation versus force is shown in Fig. 4. We determine the stiffness on the basis of the deformation versus force. The results are presented in Table I.

TABLE I

The mechanical properties of foams.

\begin{tabular}{l|c|c}
\hline \hline NVE65H & Conventional & Auxetic \\
\hline the Poisson ratio & $0.776 \pm 0.089$ & $-0.198 \pm 0.012$ \\
stiffness $[\mathrm{N} / \mu \mathrm{m}]$ & $0.011 \pm 0.001$ & $0.085 \pm 0.003$ \\
$R_{C V}$ & - & $2.7 \pm 0.1$
\end{tabular}

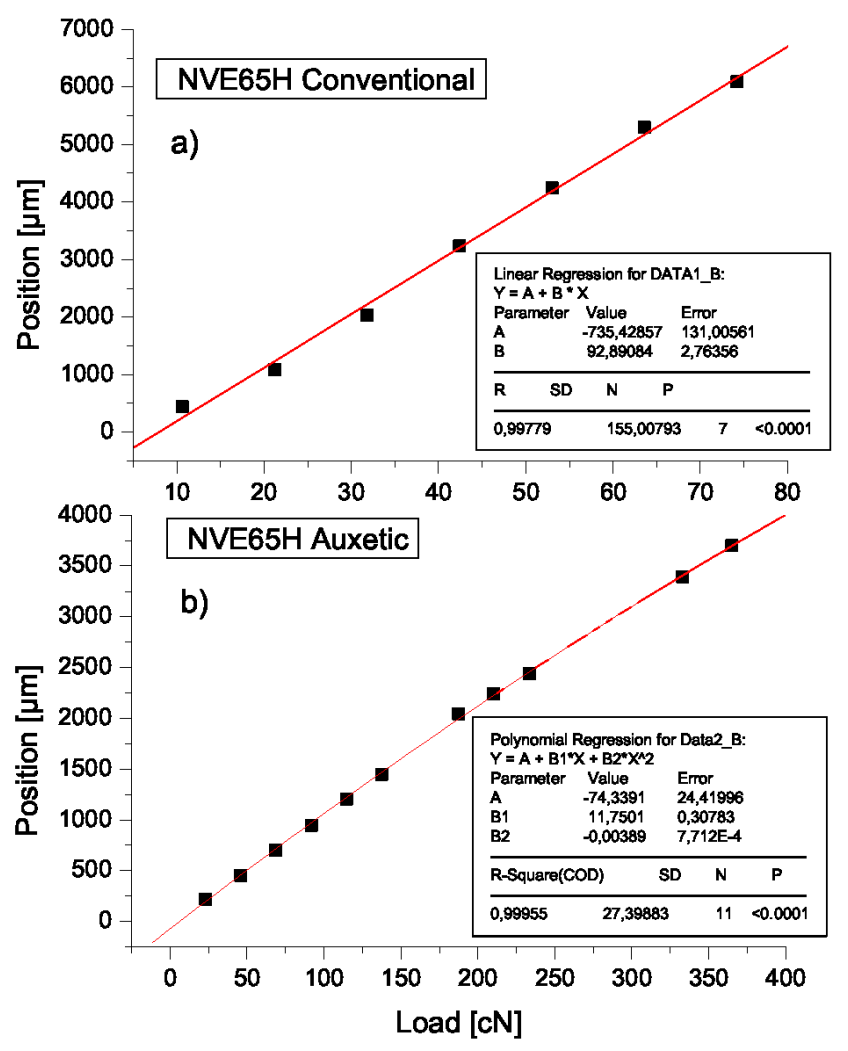

Fig. 4. Deformation versus force: (a) conventional foam, (b) auxetic foam.

TABLE II

Values of the volumetric compression ratio $\left(R_{C V}\right)$ and the Poisson ratio for the following polyurethane polyester foam [14].

\begin{tabular}{l|c|c}
\hline \hline Foam type & $R_{C V}$ & the Poisson ratio \\
\hline S364MDA & 2.0 & -0.111 \\
Lam353MDA & 2.0 & -0.088 \\
D30130A & 2.0 & -0.120 \\
S28280A & 2.2 & -0.110 \\
S28280A & 3.1 & -0.130 \\
S28280A & 4.4 & -0.040
\end{tabular}

The measurements of the Poisson ratio for viscoelastic foams like NVE65H are presented in paper [15]. A characteristic parameter of auxetic foams is the volumetric compression ratio $R_{C V}=V_{p} / V_{a}$ (where $V_{p}$ - the initial volume of the foam specimen before processing, and $V_{a}$ - the final volume of the processed foam). Other authors also make auxetic foams as illustrated in Table II. The auxetic foam NVE65H has the Poisson ratio higher than foams from Table II and the stiffness was determined for it.

\section{Conclusions}

Auxetic foams and conventional foams have quite similar properties, because of linearity of the diagrams describing a dependence of the deformation versus the 
stress. The conventional foam of a type NVE65H exhibits linearity, while an auxetic NVE65H foam exhibits a concavity. The structure of polyurethane foams are more resistant to indentations than conventional foams (Fig. 5). An auxetic material contracts laterally where material flows to the site of the impact (Fig. 6). The auxetic material is more deuce at the site of the impact and therefore it is more resistant to indentation. Analytical approaches to this type structure are difficult to find [16].
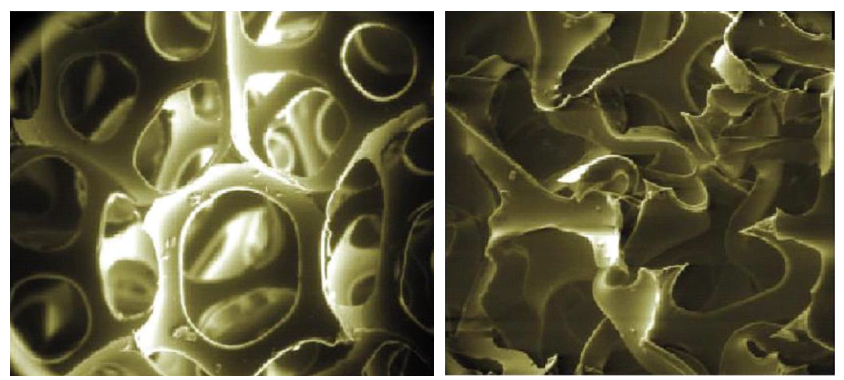

Fig. 5. Example of polyurethane foams (left: conventional and right: auxetic) [17].
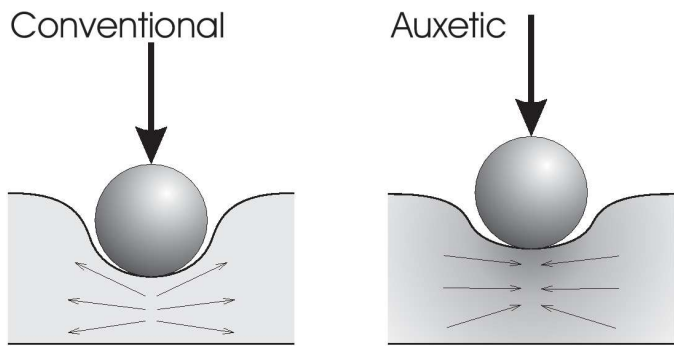

Fig. 6. Behavior of conventional and auxetic materials under impact load [18].

\section{References}

[1] R.S. Lakes, Science 235, 1038 (1987).

[2] R.S. Lakes, K.J. Elms, J. Composite Mater. 27, 1193 (1993).

[3] N. Chan, K.E. Evans, J. Mater. Sci. 32, 5725 (1997).

[4] T. Lee, R.S. Lakes, J. Mater. Sci. 32, 2397 (1997).

[5] N. Chan, K.E. Evans, J. Cell. Plast. 34, 231 (1998).

[6] C.W. Smith, F. Lehman, R.J. Wootton, K.E. Evans, Cell. Polym. 18, 79 (1999).

[7] E.A. Friis, R.S. Lakes, J.B. Park, J. Mater. Sci. 23 , 4406 (1988).

[8] C.W. Smith, J.N. Grima, K.E. Evans, Acta Mater. 48, 4349 (2000).

[9] R. Pryor, O.L. Hageniers, W.P.T. North, Appl. Opt. 11, 308 (1972).

[10] A. Wasilewski, T. Więcek, L.B. Magalas, Solid State Phenom. 89, 343 (2003).

[11] A. Wasilewski, T. Więcek, A.K. Jonscher, Opt. Appl. 29, 1 (1999).

[12] J. Goodman, Introduction to Fourier Optics, Mir, Moskva 1970

[13] L. Pekala, A. Podkoscielny, A. Wasilewski, T. Wiecek, Proc. SPIE 5484, 354 (2004).

[14] J. Lisiecki, D. Nowakowski, P. Reymer, Fatigue Aircraft Struct. 1, 72 (2014).

[15] T. Wiecek, A. Belczyk, M. Pucher, A. Wasilewski, Proc. SPIE 6937, 693721 (2007).

[16] Y. Prawoto, Computat. Mater. Sci. 58, 140 (2012).

[17] J.N. Grima, K.E. Evans, J. Mater. Sci. 41, 3193 (2006).

[18] A. Alderson, Chem. Ind. 10, 384 (1999). 